\title{
Análisis empírico de correlación entre el indicador de estructura de capital y el indicador de margen de utilidad neta en pequeñas y medianas empresas
}

\author{
BARRERA LIEVANO, JHONY AlEXANDER
}

Programa de Especialización en Gerencia de Proyectos

Facultad de Ciencias Empresariales de Uniminuto Virtual y Distancia (Colombia)

Correo electrónico: jobarrera@uniminuto.edu

PARADA FonseCA, SANDRA PATRICIA

Programa de Especialización en Gerencia de Proyectos

Facultad de Ciencias Empresariales de Uniminuto Virtual y Distancia (Colombia)

Correo electrónico: sparada@uniminuto.edu

SERRANO SERRATO, LEIDY VIVIANA

Programa de Administración Financiera

Facultad de Ciencias Empresariales de Uniminuto Virtual y Distancia (Colombia)

Correo electrónico: leydi.serrano@uniminuto.edu

\section{RESUMEN}

La presente investigación aborda los resultados financieros del año 2017 de 2.622 empresas de una ciudad capital de América Latina con los que se calculan los indicadores financieros de Estructura de capital y Margen de utilidad neta. Se plantea como objetivo central determinar si existe o no una relación directa y significativa entre los indicadores financieros mencionados a través del cálculo del coeficiente de correlación de Pearson. Se aplica una metodología cuantitativa, de tipo explicativa, que procura identificar patrones en los resultados financieros analizados que den luz de la existencia de una estructura de capital general óptima (relación entre el pasivo y el patrimonio). Como conclusión general se obtiene que, con los datos analizados, no se evidencia que exista una relación directa y significativa entre los indicadores trabajados. Queda como futuros temas de investigación realizar el mismo proceso, pero por sectores económicos y/o por tamaño de empresa.

Palabras clave: empresa, financiación, correlación.

Clasificación JEL: G32.

MSC2010: $62 J 10$. 


\title{
Empirical analysis of correlation between Debt/Equity (D/E) Ratio and the Net profit margin ratio in Small and Medium Enterprises
}

\begin{abstract}
This research approach the financial results of the year 2017 of 2,622 companies of a capital city from Latin America with these the financial indicators of Capital structure and Net profit margin are calculated. The main objective is to determine whether there is a direct and significant relationship between the aforementioned financial indicators through the calculation of the Pearson correlation coefficient. A quantitative methodology is applied, of explanatory type, which seeks to identify patterns in the analyzed financial results that give light of the existence of an optimal general capital structure (relationship between the liability and equity). As a general conclusion, it is obtained that, with the data analyzed, there is no evidence that there is a direct and significant relationship between the indicators worked on. It remains as future research topics to carry out the same process, but by economic sectors and / or by company size.
\end{abstract}

Keywords: company, financing, correlation.

JEL classification: G32.

MSC2010: 62J10.

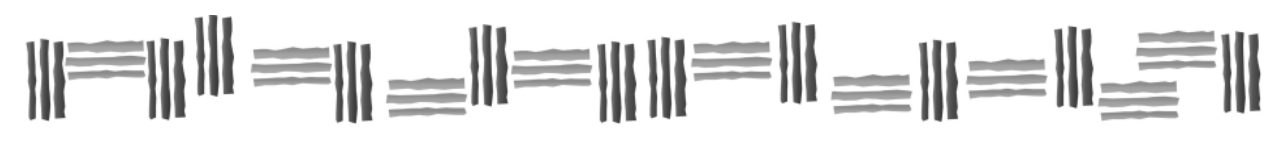




\section{Introducción.}

La estructura de financiación de la empresa ha sido un tema tratado ampliamente, que ha traído consigo el establecimiento de teorías, traducidas en modelos, sobre cómo debe configurarse, para mejorar, o explicar, los resultados financieros del ente económico, entre los que se encuentran: "Estructura de financiación", "Teoría del equilibrio estático", y la "Teoría de la jerarquía financiera". La distribución ideal de la relación deuda / patrimonio y su impacto en los resultados financieros trae consigo varias incógnitas en las que se debe reflexionar, entre otras: ¿Existe una relación directa y significativa, entre la estructura de capital de la empresa respecto al resultado financiero del ejercicio de éstas? El identificar un patrón de relación puede llevar a pensar en que hay la posibilidad de que exista una relación ideal en los componentes de financiación de la empresa (pasivo y patrimonio), de su estructura económica, lo que se traduce en el total activo.

Como objetivo general del estudio se establece determinar si existe o no una relación directa y significativa, entre la estructura de capital de la empresa respecto al resultado financiero del ejercicio de éstas. Para abordar esta pregunta, amplia en su alcance, se propone trabajar con la información financiera de las Pequeñas y Medianas empresas (PYME) de la localidad de Engativá (Bogotá DC, Colombia) que renovaron matrícula mercantil ante la Cámara de Comercio de Bogotá para el año 2018, lo cual garantiza que hayan suministrado la información financiera del año 2017, y delimita la población objeto de estudio.

Para esto, se acudió a la Cámara de Comercio de Bogotá, entidad que centraliza la información de la mayor cantidad de empresas de la ciudad y que tiene entre sus frentes de acción los registros públicos de: registro mercantil, registro único de proponentes y registro de entidades sin ánimo de lucro (Cámara de Comercio de Bogotá, 2018). La Cámara de Comercio de Bogotá suministró una base de datos con la información total de las PYME de la localidad de Engativá, 2.929 PYME, que presentaron las características descritas previamente. Entre la información que suministra dicha Cámara de Comercio se encuentra el total pasivo de cada PYME, al igual que el patrimonio, las ventas netas y la utilidad o pérdida neta, datos todos para el año 2017. Se parte del supuesto que la información financiera reportada por las empresas a la Cámara de Comercio es verídica y corresponde a la realidad, salvedad que realiza la misma entidad asegurando que no son responsables de la veracidad de la información reportada por los diferentes entes económicos. Se depura la base de datos para trabajar con la información adecuada, lo cual deja hábiles para el estudio 2.622 pequeñas y medianas empresas.

Se seleccionan dos indicadores financieros, uno de "deuda" y otro de "rendimiento": indicador de Estructura de capital (indicador de deuda), e indicador de Margen de utilidad neta (indicador de rendimiento o rentabilidad). Teniendo en cuenta la información suministrada por la Cámara de Comercio de Bogotá se calculan los resultados de dichos indicadores para cada una de las 2.622 PYME. Se toman dos indicadores que se construyen con estados financieros diferentes, por un lado, el indicador de Estructura de capital se calcula con cuentas del estado financiero de situación financiera, mientras que el indicador de Margen de utilidad neta toma cuentas provenientes del estado financiero de resultados, también llamado estado de pérdidas y ganancias. Esta selección hace que no existan de forma directa resultados secuenciales por operaciones de suma o resta entre cifras del mismo estado financiero entre los dos indicadores.

Finalmente, se determina el coeficiente de correlación de Pearson entre los resultados de las PYME para el periodo descrito, de los dos indicadores financieros definidos, se analizan los datos recolectados y los resultados obtenidos por los procedimientos anteriormente mencionados, se generan conclusiones respecto a lo hallado y se proponen nuevos interrogantes para investigaciones futuras. 


\section{Revisión literaria.}

Teniendo en cuenta la pregunta de investigación planteada en este estudio, y el objetivo principal establecido, se hace necesario entrar a abordar a nivel teórico cuatro ítems fundamentales:

i) la estructura de financiación de la empresa,

ii) los indicadores financieros de Estructura de capital y Margen de utilidad neta,

iii) el coeficiente de correlación de Pearson como herramienta estadística y

iv) a nivel legal lo que son las pequeñas y medianas empresas en Colombia.

A continuación, se desarrollan, en su orden, cada uno de estos puntos.

\subsection{Sobre la estructura de financiación.}

Las empresas son vitales para la salud de la economía. Visto desde el circuito económico, las relaciones que generan las empresas con la sociedad, puntualmente con las familias, enmarcan la prestación de servicios y la oferta de bienes, además de la generación de puestos de trabajo (Massad, 2007). Desde la teoría económica, como dice O'Kean (2013), Adam Smith plantea como base del proceso productivo a los medios de producción, a saber, capital, tierra y trabajo. El capital, por su parte, puede venir de varias fuentes, sin embargo, visto desde el estado financiero de situación financiera solo viene de dos fuentes categóricas, ya que "El reconocimiento contable del activo implica también reconocimiento simultaneo de un pasivo, (...) u otro incremento en el patrimonio neto" (Fernández, 2014, p. 63). Desde este punto de partida, la financiación de una empresa puede venir de: pasivos y/o patrimonio.

Las decisiones de financiación de las empresas en general, según se puede apreciar en la literatura existente, se asocian a diferentes factores, entre otros, el tamaño de la empresa (Botello, 2015), los rasgos característicos de las personas que ejercen la gerencia de la empresa (Barton \& Gordon, 1987, citados por Pesce et al., 2015) y el impacto de los costos de agencia en la adquisición de deuda (Pesce et al., 2015), sea por costo o por beneficio fiscal de la deuda. Las principales teorías o planteamientos, de mayor reconocimiento, que generaron modelos ampliamente debatidos respecto a la estructura de capital de la empresa son, entre otros planteamientos: Estructura de financiación de la empresa (Modigliani \& Miller, 1958) (Modigliani \& Miller, 1963), Teoría del Equilibrio Estático (Myers, 1977) y Teoría de la jerarquía financiera (Donaldson, 1961; Myers, 1984; Myers \& Majluf, 1984), los cuales se abordan a continuación.

\subsubsection{Estructura de financiación}

El análisis de la estructura de financiación de la empresa fue abordado inicialmente por Modigliani y Miller (1958) en su documento The cost of capital, corporation finance and the theory of investment, en donde plantean un modelo muy limitado, frente a las restricciones planteadas en el mismo, como lo fueron un estado de competencia atomizado, o la facilidad de acceso a los mercados de un grupo de empresas relativamente pequeño, que en palabras de los mismos autores fueron simplificaciones necesarias para abordar su planteamiento del problema en absoluto. Barona-Zuluaga y Rivera-Godoy (2012) frente al modelo planteado por Modigliani y Miller (1958) mencionan que una de las conclusiones que deja el planteamiento teórico es que la estructura de financiación no afecta el valor de la empresa. Frente a su propio modelo, Modigliani y Miller (1963) refutan y corrigen en su artículo Corporate Income Taxes and the Cost of Capital: A Correction, los planteamientos matemáticos y supuestos de su modelo, documento en que los autores mencionan en su primer párrafo: "El propósito de esta comunicación es corregir un error en nuestro documento "El costo del capital, el financiamiento de la corporación y la teoría de la inversión" (esta revisión, junio de 1958)" (p.433). La corrección se centró en las fórmulas planteadas por los autores, los supuestos, lo cual llevó a los autores a la estimación de las ventajas fiscales del financiamiento de deuda en el modelo planteado como ellos 
mismos lo concluyen. De igual forma concluyen que "la existencia de una ventaja impositiva para el financiamiento de la deuda no significa necesariamente que las empresas deben en todo momento buscar usar la cantidad máxima posible de deuda en su estructura de capital" (p.442).

\subsubsection{Teoría del Equilibrio Estático (Trade-off Theory)}

Tiene como punto de partida el documento de Myers (1977), que estipuló que el monto de la deuda emitida por la empresa debe ser igual al valor presente de las opciones de la empresa para realizar inversiones futuras, esa cantidad es la que maximiza el valor de mercado de la empresa. Para este caso se racionaliza la inversión en activos de acuerdo al beneficio óptimo que se puede tener de acuerdo a la fuente de financiación que se tome, no siendo exclusivamente la mejor opción la adquisición de deuda.

De acuerdo a lo mencionado por Mondragón-Hernández (2011): "Para la teoría del trade off, el valor de la firma no está dado por el rendimiento de los activos presentes de la empresa, sino por el valor presente neto del rendimiento que podría obtener con futuras inversiones" (p.172). Lo anterior conllevará a que la empresa cuente con una estructura de capital adecuada que le permita maniobrar tanto en el presente como en el futuro, en otras palabras, que no comprometa de manera negativa sus posibilidades futuras de inversión. Esto lleva a que la empresa emplee el balance adecuado entre deuda y capital, de ahí el nombre de teoría del equilibrio estático (trade-off Theory).

\subsubsection{Teoría de la jerarquía financiera}

Expuesta por los planteamientos del modelo planteado por Myers y Majluf (1984) concluye que los empresarios, cuando tienen "información superior", se inclinarán por fuentes de financiación internas sobre las externas. Esto debido a varios factores, entre los cuales se encuentran el costo de financiación y la equidad (participación) en la propiedad de la empresa.

Pesce et al. (2015), citando a Donaldson (1961), Myers (1984), Myers y Majluf (1984), refieren la jerarquía de preferencias con el siguiente orden: primero, son los fondos generados internamente; segundo, deuda externa; tercero, ampliación de capital social, lo que se entenderá como emisión de acciones. Según lo anterior, y como lo refiere Mondragón-Hernández (2011) "supone que la empresa no tiene una estructura óptima de capital" (p.172), dejando de lado aún los beneficios tributarios que existen por contraer deuda.

\subsection{Indicadores financieros de Estructura de capital y Margen de utilidad neta.}

Los indicadores financieros son base para evaluar y analizar en términos relativos los resultados financieros obtenidos por la empresa en un periodo determinado (Gitman \& Zutter, 2016). Como lo menciona Fontalvo, De la Hoz y Vergara (2012): "Los indicadores financieros son el producto de establecer resultados numéricos basados en relacionar dos cifras o cuentas bien sea del Balance General y/o del Estado de Pérdidas y Ganancias" (p.321). Ahora bien, los indicadores financieros pueden ser clasificados según el origen de las cifras numéricas a relacionar, en cinco categorías, a saber: "de liquidez, de actividad, de deuda, de rentabilidad y de mercado" (Gitman \& Zutter, 2016, p. 72). Para este caso se abordarán el indicador financiero de estructura de capital, que es un indicador de deuda, y el indicador financiero de margen de utilidad neta, que es un indicador de rentabilidad o rendimiento.

\subsubsection{Indicador financiero de Estructura de capital}

El indicador financiero de estructura de capital hace parte del grupo de indicadores de deuda. Como lo menciona Córdoba (2014), el resultado de dividir el valor del total pasivo entre el valor del total patrimonio da como resultado la estructura de capital. 
Tabla 1. Indicador de Estructura de capital.

\begin{tabular}{|l|c|}
\hline \multicolumn{1}{|c|}{ Nombre del indicador } & Fórmula \\
\hline Estructura de capital & $=$ (Total pasivo / Total patrimonio) \\
\hline
\end{tabular}

Fuente: Adaptado de Córdoba (2014).

Según la relación matemática de las variables enunciadas en la fórmula, se puede inferir que la interpretación del resultado obtenido puede ser que por cada unidad que hay en la cuenta del denominador existen $x$ cantidad de unidades en la cuenta del numerador. En otras palabras, que por cada unidad monetaria que hay en el total patrimonio, existen $x$ cantidades de unidades monetarias en el total pasivo, lo que significaría para el caso de este indicador financiero que, por cada peso que existe en el patrimonio, la empresa cuenta con $x$ cantidad de pesos adeudados, $o$ que, por cada peso que los accionistas tienen en el patrimonio de la empresa, la empresa se financia con $x$ cantidad de pesos. En todos los casos $x$ es igual al resultado de la operación de la fórmula del indicador.

\subsubsection{Indicador financiero de Margen de utilidad neta}

El indicador financiero de margen de utilidad neta hace parte del grupo de indicadores de rentabilidad (también llamado grupo de indicadores de rendimiento). Como lo menciona Córdoba (2014) este indicador también se conoce como "índice de productividad". Este indicador es el resultado de dividir el valor de la utilidad neta entre el total de las ventas netas.

Tabla 2. Indicador de Margen de utilidad neta.

\begin{tabular}{|c|c|}
\hline Nombre del indicador & Fórmula \\
\hline Margen de utilidad neta & $=($ Utilidad neta / Ventas netas $)$ \\
\hline
\end{tabular}

Fuente: Adaptado de Córdoba (2014).

Al igual que con el anterior indicador, según la relación matemática de las variables enunciadas en la fórmula, se puede inferir que la interpretación del resultado obtenido puede ser que por cada unidad que hay en la cuenta del denominador existen $x$ cantidad de unidades en la cuenta del numerador. En otras palabras, que por cada unidad monetaria que resultado de las ventas netas, existen $x$ cantidades de unidades monetarias que se convirtieron en utilidad neta, lo que significaría para el caso de este indicador financiero que, por cada peso de ventas netas, la empresa obtuvo $x$ cantidad de pesos como utilidad neta. En todos los casos $x$ es igual al resultado de la operación de la fórmula del indicador.

\subsection{Coeficiente de correlación de Pearson.}

El coeficiente de correlación, coeficiente de correlación lineal, o coeficiente de correlación de Pearson, determina el grado de relación o correlación que puede haber entre dos variables y se ha definido como la raíz cuadrada del coeficiente de correlación al cuadrado o coeficiente de determinación. Básicamente el coeficiente de correlación arroja un resultado que se encuentra entre el intervalo de -1 a 1 . Si su resultado es positivo significa que existe una relación directa entre las dos variables y si su resultado es negativo significa que existe una relación inversa entre las dos variables. La relación, directa o inversa, será más fuerte si el resultado está más cerca al 1 ó -1 respectivamente (resultado más alejado del 0) (Martinez \& Levin, 2011). 


\subsection{Sobre la pequeña y mediana empresa - PYME.}

En el año 2000 Colombia a través de la ley 590 establece un marco de fomento a las micro, pequeñas y medianas empresas en todo el territorio nacional. Como es de esperar, esta ley contempla una serie de mandatos para el fomento de la creación y el crecimiento de este tipo de empresas, que también tipificó de acuerdo a unas características especiales: número de trabajadores y total de activos.

Posterior a la ley 590 de 2000, aparece la ley 905 de 2004 que incorpora aclaraciones frente a lo que se puede entender respecto a una micro, pequeña o mediana empresa. Tres años más tarde aparece la ley 1151 de 2007 que amplía la aclaración frente a lo que se puede entender respecto a una micro, pequeña o mediana empresa e incorpora una nueva variable característica para la tipificación de cada nivel (micro, pequeña, mediana), además de una relación entre las mismas (Nieto et al., 2015). Finalmente, la ley 1450 de 2011 define el concepto de empresa y especifica los criterios para determinar el tamaño de la empresa, haciendo separación entre estos.

Según lo expuesto por la legislación vigente (Tabla 3), una PYME se clasifica de acuerdo a los siguientes criterios:

a) Planta de personal entre once y doscientos trabajadores, o

b) Activos totales por valor entre quinientos uno a treinta mil salarios mínimos mensuales legales vigentes (smmlv).

Respecto a la tercera variable tenida en cuenta desde la ley 1151 de 2007, que fue ratificada, y a la vez separada, en la ley 1450 de 2011, "Valor de ventas brutas anuales", Nieto et al. (2015) especifica que, puntualmente a 2015 la nueva clasificación del 2011 aún no había sido reglamentada, lo cual es vigente hasta hoy, pues no existe reglamentación para ésta.

\section{Contextualización.}

Ubicada al noroccidente de la ciudad de Bogotá DC, la localidad de Engativá cuenta con una extensión de total de 3.588,1 hectáreas (Secretaría de Planeación Distrital, 2011, p. 7), unos 35,88 kilómetros cuadrados, casi el 1,01\% del total de la extensión del área metropolitana de la ciudad, que según Peña-Barrera (2010, p. 35) es de 3.550 kilómetros cuadrados. En su estructura interna, la localidad de Engativá está integrada por nueve Unidades de Planeamiento zonal (UPZ): 26 Las Ferias, 29 Minuto de Dios, 30 Boyacá Real, 31 Santa Cecilia, 72 Bolivia, 73 Garcés Navas, 74 Engativá, 105 Jardín Botánico y 116 Álamos (Decreto 619 de 2000), 24 zonas postales (Secretaría de Planeación Distrital, 2007, p.26), que en total se estima alberga 883.319 habitantes (Secretaría de Planeación Distrital, 2018a).

Del total de la población de la localidad, a 2014, según datos del Departamento Administrativo Nacional de Estadística (DANE) citados por el Consejo Local de Gestión de Riesgos y Cambio Climático (2017), Engativá contó con un total del $9 \%$ de su población por debajo de la línea de pobreza, y un 3,2\% en pobreza extrema, mientras que el desempleo estuvo en el $13,5 \%$.

A 2018, primer trimestre, según datos de la Secretaría de Planeación Distrital de la ciudad de Bogotá, la localidad de Engativá concentró el 9,5\% (71.895) del parque empresarial de la ciudad de Bogotá, que estuvo compuesto al primer trimestre de 2018 por 757.782 empresas con matrícula mercantil activa en la Cámara de Comercio de Bogotá (Secretaría de Planeación Distrital, 2018b). 
Tabla 3. Clasificación PYME según la legislación de Colombia.

\begin{tabular}{|c|c|c|c|c|c|}
\hline 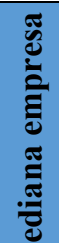 & 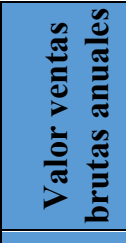 & 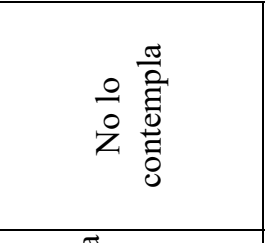 & 을 & 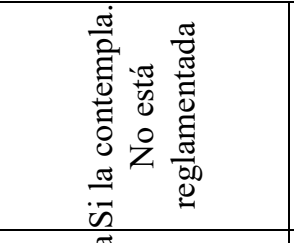 & 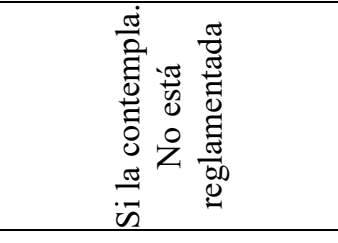 \\
\hline 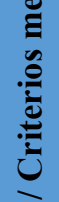 & 苋 & 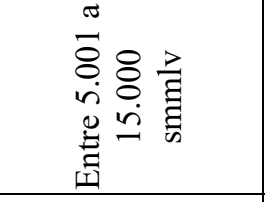 & 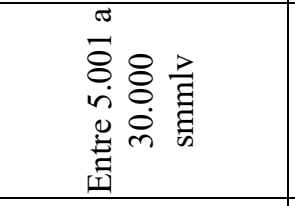 & 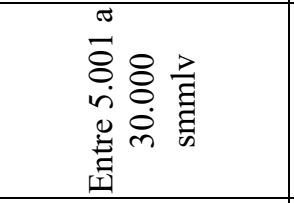 & 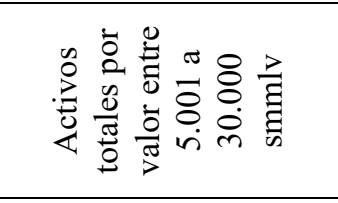 \\
\hline לֶ. & 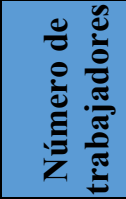 & 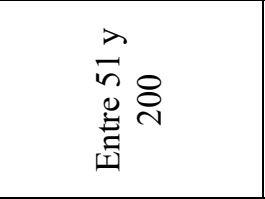 & 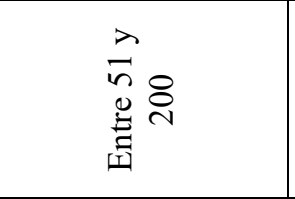 & 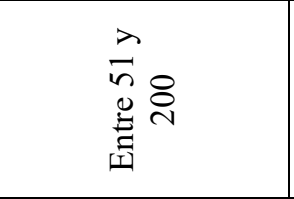 & $\begin{array}{l}\lambda \\
\vec{n} \\
\stackrel{\Xi}{\Xi} \\
\text { 芒 }\end{array}$ \\
\hline 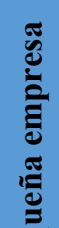 & 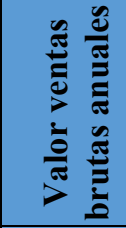 & $\frac{\stackrel{\pi}{0}}{\stackrel{2}{0}}$ & 을 & 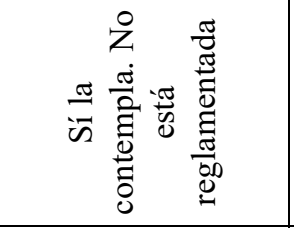 & 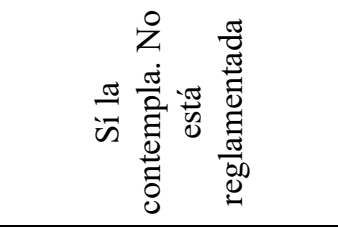 \\
\hline 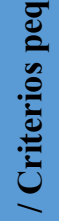 & 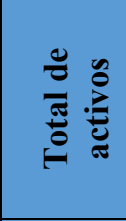 & 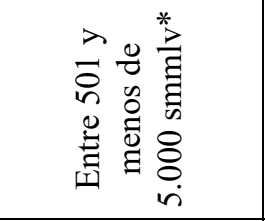 & 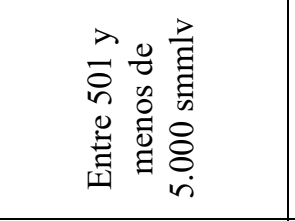 & 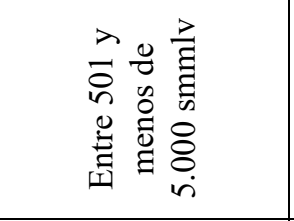 & 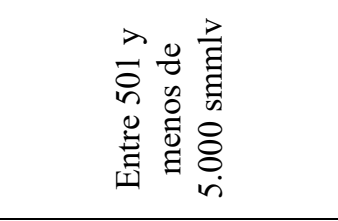 \\
\hline 离 & 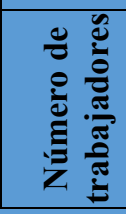 & 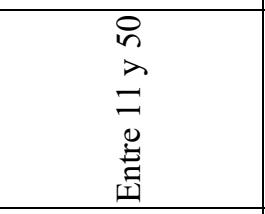 & 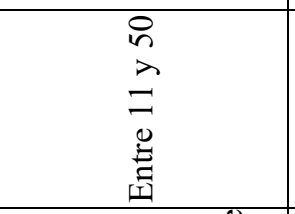 & 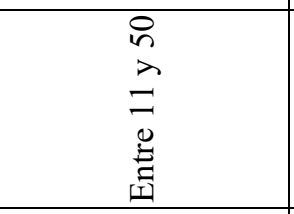 & 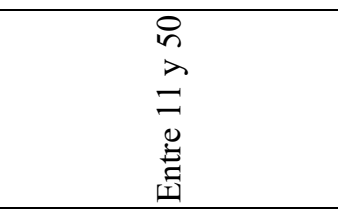 \\
\hline & 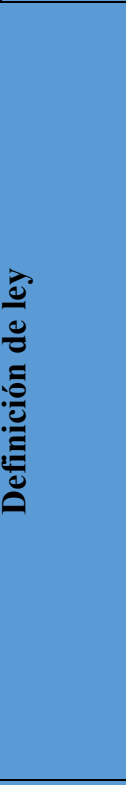 & 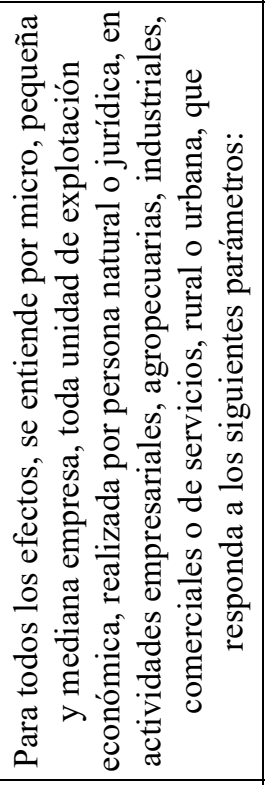 & 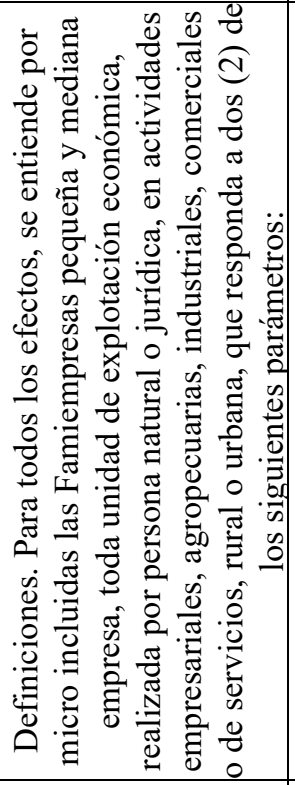 & 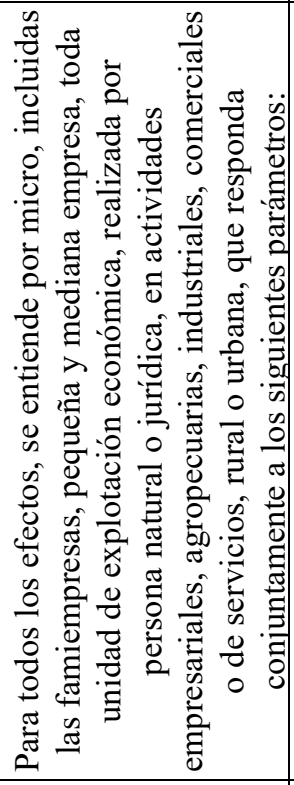 & 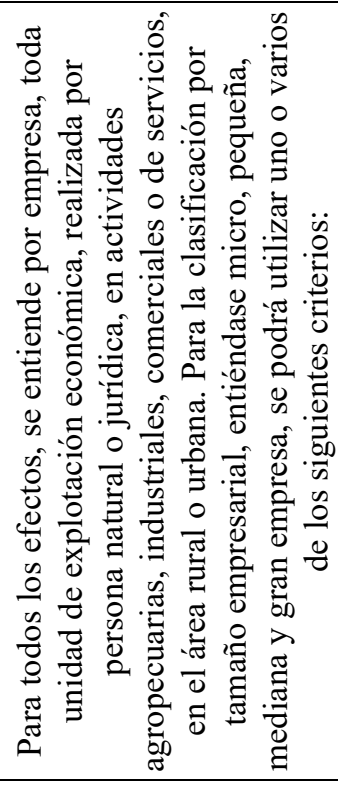 \\
\hline & ङ્త & 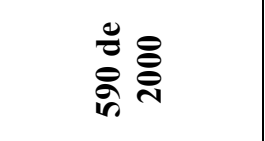 & 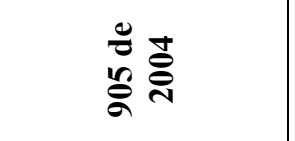 & $\stackrel{\overbrace{}}{\tilde{\sigma}}$ & 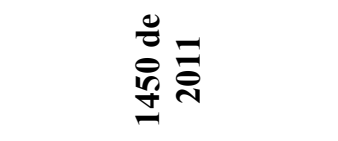 \\
\hline
\end{tabular}

* smmlv: salarios mínimos mensuales legales vigentes.

Fuente: Adaptado de: Ley 590 de 2000, Ley 905 de 2004, Ley 1151 de 2007 y Ley 1450 de 2011. 
Según la base de datos suministrada por la Cámara de Comercio de Bogotá, la localidad de Engativá cuenta con 2.482 pequeñas empresas y 447 medianas empresas, para un total de 2.929 pequeñas y medianas empresas - PYME con matrícula mercantil activa a julio de 2.018, con las siguientes clasificaciones: del 100\% de las PYME de la localidad de Engativá el 15,3\% son medianas empresas y el $84,7 \%$ son pequeñas empresas, presentando una relación de 5,5 pequeñas empresas por cada mediana empresa registrada con matrícula activa a 2018, y que reportaron estados financieros del año 2017.

Tabla 4. PYME localidad de Engativá - Matricula activa 2018.

\begin{tabular}{|c|c|c|}
\hline Tipo de sociedad & Cantidad & Participación \\
\hline Mediana & 447 & $15,3 \%$ \\
\hline Pequeña & 2.482 & $84,7 \%$ \\
\hline Total general & $\mathbf{2 . 9 2 9}$ & $\mathbf{1 0 0 \%}$ \\
\hline
\end{tabular}

Fuente: Adaptado de Cámara de Comercio de Bogotá (2018).

Según el tipo de sociedad, se identificó que, del total de las PYME de la localidad de Engativá, la mayor concentración se encuentra en el tipo de sociedad "sociedad por acciones simplificada (S.A.S.)", con una participación del 64,0\%, seguida de la "Sociedad limitada (Ltda.)" con un 19,6\%. El tipo de sociedad con menor participación es la "comandita por acciones (S.C.A.)", 0,1\%, seguida de la "empresa unipersonal" con un $1,0 \%$.

Tabla 5. PYME localidad de Engativá por tipo de sociedad - Matricula activa 2018.

\begin{tabular}{|c|c|c|}
\hline Tipo de sociedad & Cantidad & Participación \\
\hline Anónima & 145 & $5,0 \%$ \\
\hline Comandita por acciones & 4 & $0,1 \%$ \\
\hline Comandita Simple & 33 & $1,1 \%$ \\
\hline Empresa unipersonal & 29 & $1,0 \%$ \\
\hline Extranjera & 20 & $0,7 \%$ \\
\hline Limitada & 573 & $19,6 \%$ \\
\hline Persona Natural & 249 & $8,5 \%$ \\
\hline Sociedad por Acciones Simplificada & 1.876 & $64,0 \%$ \\
\hline Total general & $\mathbf{2 . 9 2 9}$ & $\mathbf{1 0 0} \%$ \\
\hline
\end{tabular}

Fuente: Adaptado de Cámara de Comercio de Bogotá (2018).

El parque PYME de la localidad cuenta con participación en 18 sectores económicos, presentando su mayor concentración, en orden de mayor a menor, en los sectores económicos de Comercio al por mayor y al por menor reparación de vehículos automotores y motocicletas; Industrias manufactureras; y, Construcción, con una participación general superior al 60\%.

\section{Metodología del estudio.}

Con el objetivo de dar respuesta a la pregunta principal de este estudio, y abordar lo planteado como objetivo general, se desarrolla una investigación con metodología cuantitativa, de tipo explicativa, que busca identificar patrones en los resultados financieros obtenidos por las PYME de la localidad de Engativá para el año 2017. La fuente principal de datos es la información suministrada por la Cámara de Comercio de Bogotá respecto al total de pequeñas y medianas empresas - PYME que presentan matrícula mercantil activa para el año 2018, y que reportaron su información financiera del año 2017, por lo cual se constituye como una fuente secundaria. El diseño de investigación se cataloga como documental. 
La información que entrega la Cámara de Comercio de Bogotá presenta cifras financieras agregadas como se relaciona a continuación: activo total; activo corriente; total pasivo; pasivo corriente; obligaciones largo plazo; patrimonio; ventas netas; costo de ventas; gastos de administración; utilidad o pérdida operacional; utilidad o pérdida neta. La base de datos se depura teniendo en cuenta la siguiente variable: se seleccionan todas las PYME que presentan ventas en el periodo 2017.

Partiendo de la información relacionada, se procede a calcular, para cada PYME, los indicadores financieros de Estructura de capital y Margen de utilidad neta, que se analizan contrastando los resultados obtenidos por las PYME objeto del estudio, según el coeficiente de correlación de Pearson que presenten los resultados de los dos indicadores. Para la organización y el procesamiento de datos se utiliza el programa MS Excel 2016.

En resumen: Población, PYME de la localidad de Engativá; característica propia de la población, quienes renovaron matrícula mercantil para el año 2018; unidad de tiempo a analizar, año 2017; muestra, no se considera una muestra, se trabaja con el total de la población que presente la información financiera de funcionamiento en el año 2017 y que presentan ventas para este mismo periodo; fuente de información, Cámara de Comercio de Bogotá; tipo de fuente, secundaria; tipo de investigación, cuantitativa y documental de tipo explicativa; programas empleados para la organización y procesamiento de datos, MS Excel 2016; análisis de los datos, indicador de Estructura de capital e indicador de Margen de utilidad neta, teniendo en cuenta el coeficiente de correlación de Pearson que presenten los resultados de los dos indicadores.

\section{Resultados.}

La localidad de Engativá a julio de 2018 contó con un total de 2.929 PYME con matrícula mercantil activa en la Cámara de Comercio de Bogotá. De las 2.929 PYME, 447 son medianas empresas y 2.482 son pequeñas empresas.

Del total relacionado, se identificó que 307 PYME no reportaron ventas para el año 2017 (282 pequeñas empresas y 25 medianas empresas). De las 307 PYME, 21 presentan fecha de matrícula mercantil en el año 2018 (20 pequeñas empresas y una mediana empresa) motivo por el cual no pueden reportar información de años anteriores. El restante de PYME, 286, se asume que no presentaron actividad mercantil para el año 2017, aunque algunas de éstas reportan utilidad en el ejercicio de dicho periodo. Para este caso las 307 PYME que no presentan ventas en el periodo 2017 se sacaron de la base relacionada para este estudio.

De las 2.622 PYME que presentan ventas para el año 2017 (2.200 pequeñas empresas y 422 medianas empresas), 40 no reportan patrimonio (32 pequeñas empresas y 8 medianas empresas), sin embargo, al verificar la ecuación contable de la información que hace parte del estado de situación financiera, Activo = Pasivo + Patrimonio (Díaz \& Horngren, 2011, p. 102), se verificó que al restarle al "total activo" el valor resultante de la suma de el "total pasivo" con el "patrimonio", el resultado fuera igual a 0 . Resultado que se obtuvo al verificar las $2.622 \mathrm{PYME}$, incluyendo las 40 previamente relacionadas. Para el cálculo del indicador de estructura de capital se reportará para las 40 PYME relacionadas con valor " 0 ".

Depurada la base de datos, se procede calcular la media de los resultados conjuntos de las PYME para el año 2017 y su desviación estándar, así como a expresar gráficamente los resultados conjuntos (diagrama de dispersión), para cada indicador financiero. A continuación, se presentan los resultados para cada indicador financiero. 


\subsection{Indicador de Estructura de capital.}

Al tomar los datos financieros de todas las pequeñas y medianas empresas - PYME de la localidad de Engativá, según las especificaciones mencionadas previamente, se procedió a calcular el indicador financiero de Estructura de capital con base a la fórmula especificada en la Tabla 1. Con los 2.622 resultados se calculó la media y la desviación estándar de la media (Tabla 6).

Tabla 6. Resultados media y desviación estándar del indicador de Estructura de capital 2017 - PYME localidad de Engativá.

\begin{tabular}{|c|c|}
\hline Medida & Resultado \\
\hline Media & 2,26 \\
\hline Desviación estándar & 9,90 \\
\hline
\end{tabular}

Nota: Para el cálculo de la media no se tienen en cuenta los siguientes resultados del indicador de dos medianas empresas: 430,74, y 1.108.208,86. El resultado de la media con dichos valores es el siguiente: 425,08. El resultado de la desviación estándar es el siguiente: 21634,22.

Fuente: Elaboración propia.

Como se pudo evidenciar, la media aritmética del total de resultados del indicador de Estructura de capital obtenido por las PYME de la localidad de Engativá, para el año 2017, que tuvieron registro mercantil activo a julio de 2018, y que reportaron valores en ventas en sus estados financieros, fue de 2,26, lo que significa que, en promedio, las PYME descritas, por cada peso que aportan sus propietarios hay 2,26 pesos financiados por acreedores, para el año 2017. Este resultado presenta una desviación estándar de 9,90 pesos, que, como medida de dispersión, refleja que el resultado obtenido por la media, en promedio, puede moverse 9,90 de manera positiva o negativa, en otras palabras, que en promedio el resultado del indicador de Estructura de capital para esta población está entre 12,16 y -7,64. Este resultado presenta una dispersión alta, que con respecto a la media es casi 4,4 veces su valor.

Teniendo como base el cálculo del indicador de Estructura de capital para las PYME de la localidad de Engativá, en los resultados financieros del año 2017, a continuación, se presentan de manera gráfica los resultados de las 2.622 PYME.

Gráfico 1. Resultado indicador Estructura de capital año 2017 PYME localidad de Engativá.

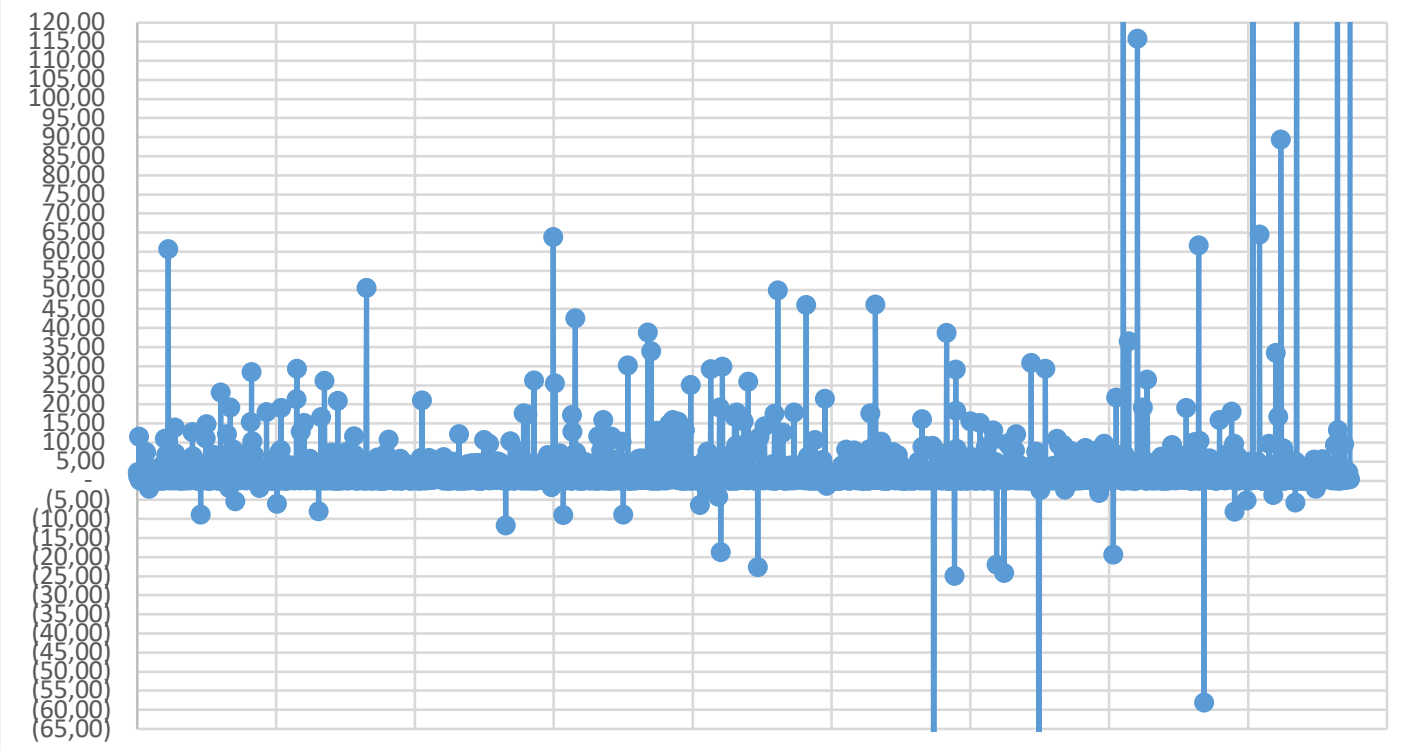

Fuente: Elaboración propia. 
Como se observa en el gráfico 1, así como en el resultado de la media y la desviación estándar de la misma, la agrupación de los resultados se encuentra de manera representativa entre los valores de 12,16 y -7,64 (2.520 PYME, el 96,10\% del total de la población), presentando la mayor concentración entre los valores de 0 y el 3: un total de 2.154 PYME ubican su resultado en este intervalo, lo que representa el $82,15 \%$.

\subsection{Indicador de Margen de utilidad neta.}

Respecto al indicador de Margen de utilidad neta, se procedió a calcular el resultado para cada una de las pequeñas y medianas empresas - PYME de la localidad de Engativá, según las especificaciones mencionadas previamente, utilizando la fórmula especificada en la Tabla 2. Con los 2.622 resultados se calculó la media, la desviación estándar de la media, y la mediana (Tabla 7).

Tabla 7. Resultados media y desviación estándar de la media del indicador de Margen de utilidad neta 2017 - PYME localidad de Engativá.

\begin{tabular}{|c|c|}
\hline Medida & Resultado \\
\hline Media & 0,28 \\
\hline Desviación estándar & 10,83 \\
\hline
\end{tabular}

Nota: Para el cálculo de la media no se tienen en cuenta los siguientes resultados del indicador de dos medianas empresas: -452,7, y -19.449,75. El resultado de la media con dichos valores es el siguiente: $-7,31$. El resultado de la desviación estándar es el siguiente: 380,02 .

$$
\text { Fuente: Elaboración propia. }
$$

Los resultados arrojados para la media aritmética para el indicador de Margen de utilidad neta obtenido por las PYME de la localidad de Engativá, para el año 2017, que tuvieron registro mercantil activo a julio de 2018, y que reportaron valores en ventas en sus estados financieros, fue de 0,28 , lo que significa que, en promedio, estas PYME para el periodo específico por cada peso en ventas netas que tuvo las PYME, en promedio, 0,28 pesos se convirtieron en utilidad neta, lo que significa que del 100\% de las ventas netas, en promedio, el $28 \%$ se convirtió en utilidad neta, para el año 2017. Sin embargo, este indicador, del promedio de las PYME, presenta una desviación estándar de 10,83 pesos, que, como medida de dispersión, refleja que el resultado obtenido por la media, en promedio, puede moverse de manera positiva o negativa, 10,83; en otras palabras, que en promedio el resultado del indicador de Margen de utilidad neta para esta población está entre 11,11 y -10,55. Este resultado presenta una dispersión alta, que con respecto a la media es casi 38,7 veces su valor.

Teniendo como base el cálculo del indicador de Margen de utilidad neta para las PYME de la localidad de Engativá, en los resultados financieros del año 2017, a continuación, se presenta de manera gráfica los resultados de las 2.622 PYME (Gráfico 2).

Como se puede evidenciar en el Gráfico 2, así como en el resultado de la media y la desviación estándar de la misma, la agrupación de los resultados se encuentra de manera representativa entre los valores de 11,11 y -10,55 (2.609 PYME, el 99,5\% del total de la población), presentando la mayor concentración entre los valores de $-0,1$ y 0,2 , un total de 2.256 PYME ubican su resultado en este intervalo, lo que representa el $86,04 \%$. 
Gráfico 2. Resultado indicador Margen de utilidad neta año 2017 PYME localidad de Engativá.

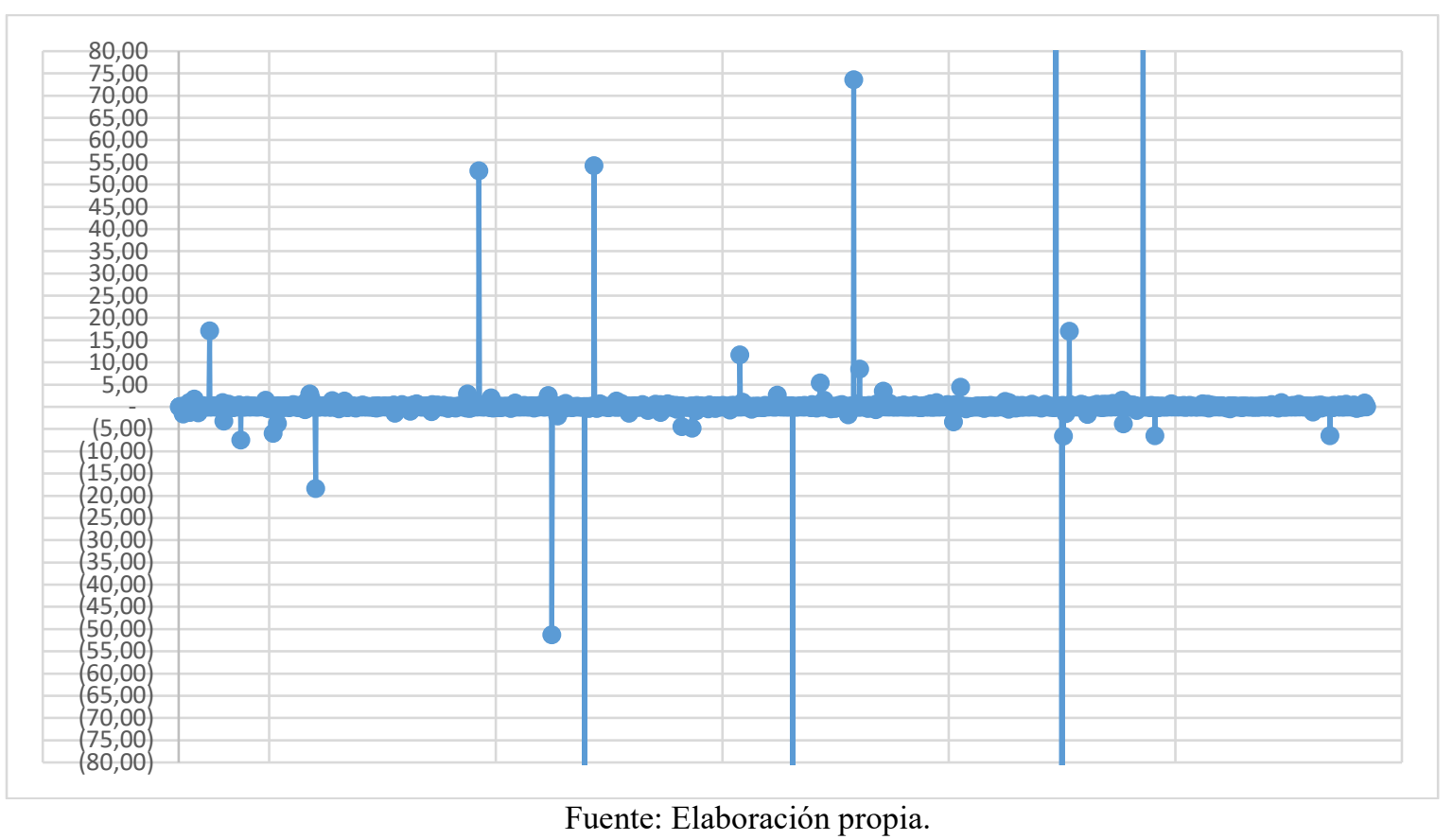

\subsection{Coeficiente de correlación de Pearson entre el indicador de Estructura de capital y el indicador de Margen de utilidad neta.}

Calculados los indicadores financieros de Estructura de capital (cuyos datos provienen directamente del Estado de situación financiera) y Margen de utilidad neta (cuyos datos provienen directamente del Estado de resultados), para todas las PYME de la localidad de Engativá, después de depurar la base de datos suministrada por la Cámara de Comercio de Bogotá, se contó para cada indicador con un total de 2.622 datos. Para el cálculo de coeficiente de correlación de Pearson entre los dos indicadores se utilizó el programa MS 2016, dentro de las "Herramientas de análisis de datos", la función para análisis de "Coeficiente de correlación", donde se seleccionaron las dos columnas donde aparecían los siguientes datos: columna 1 "indicador de Estructura de capital"; columna 2 "indicador de Margen de utilidad neta". Los resultados aparecen en la siguiente tabla.

Tabla 8. Resultado coeficiente de correlación de Pearson indicadores financieros de Estructura de capital y Margen de utilidad neta año 2017 PYME, localidad de Engativá.

\begin{tabular}{|l|r|r|}
\hline & Columna 1 & Columna 2 \\
\hline Columna 1 & 1 & \\
\hline Columna 2 & 0,00052299 & 1 \\
\hline \multicolumn{2}{|c|}{ Fuente: Elaboración propia. }
\end{tabular}

El resultado denota una relación directa de cero con cincuenta y dos mil doscientas noventa y nueve cienmillonésimas.

\section{Discusión.}

Al 31 de julio de 2018, 2.929 PYME contaron con matrícula mercantil activa en la Cámara de Comercio de Bogotá, información que fue suministrada por dicha entidad. Con la base de datos se realizó un proceso de depuración de información, teniendo presente los indicadores financieros que se construirían y la información financiera reportadas por las PYME a la cámara de comercio. La depuración de información llevó a no trabajar con la información de las PYME que no reportaron información de 
ventas para el año 2017, asumiendo que no realizaron actividades mercantiles. Esta depuración dejó hábiles para el estudio a 2.622 PYME que contaron con la información mínima y necesaria para la realización del proceso de construcción de indicadores financieros.

Debido a las grandes diferencias entre rangos de total activo que se puede presentar, por las clasificaciones especificadas por la legislación colombiana (ver Tabla 3), no fue posible trabajar con magnitudes absolutas, pero sí con magnitudes relativas, que reflejan la relación de dos magnitudes absolutas, evidenciando la comparación de una con respecto a la otra.

Los resultados obtenidos de manera general permiten evidenciar, para el indicador de Estructura de capital, una media aritmética de 2,26, con una alta desviación estándar, 9,90, presentando una alta dispersión. La mayor cantidad de resultados obtenidos por las PYME objeto de estudio estuvieron entre los valores de 0 y 3 (ver gráfico 1), más del $82 \%$ del total, el restante estuvo fuera de este rango, en ocasiones siendo más de 50 veces el rango mayor, y en otras siendo 50 veces menor (ubicando los valores en una recta numérica).

Respecto al indicador de Margen de utilidad neta, presentó una media de 0,28 con una desviación estándar de 10,83, al igual que el indicador de Estructura de capital, presentó una alta dispersión. La mayor cantidad de resultados obtenidos por las PYME objeto de estudio estuvieron entre los valores de $-0,1$ y 0,2 (ver gráfico 2), más del $86 \%$ del total; el restante estuvo fuera de este rango.

En primera instancia se evidencia que una característica propia del comportamiento de las PYME, respecto a sus resultados conjuntos presentan agrupaciones de resultados representativas: más del $82 \%$ en el indicador de Estructura de capital se ubicaron entre el rango de 0 a 3, y en el indicador de Margen de utilidad neta más del $86 \%$ entre el rango de -0.1 y 0.2 , lo que podría sugerir, en primera instancia, que sí puede existir una correlación entre los resultados de estas PYME para los indicadores de Estructura de capital y Margen de utilidad neta, lo que podría llevar a ahondar en qué rangos de Estructura de capital llevan a que exista un Margen de utilidad neta favorable para las PYME.

Encontrado lo anterior, al calcular el coeficiente de correlación de Pearson entre los resultados de los indicadores de Estructura de capital y Margen de utilidad neta, se obtuvo como resultado una correlación de 0,00052299 (cero con cincuenta y dos mil doscientas noventa y nueve cienmillonésimas) que, aunque es positiva (lo cual demuestra una relación directa entre los dos indicadores), es completamente insignificante teniendo en cuenta que la relación entre las dos variables analizadas será fuerte cuanto más se acerque al valor de 1 , lo que no sucede en este caso.

Según lo relacionado por las teorías referenciadas, éstas se enfocan en el impacto de la estructura de financiación frente al valor de la empresa, caso de lo planteado por Modigliani y Miller $(1958 ; 1963)$ y por Myers (1977), y el impacto de la estructura de financiación frente a los costos de agencia y la participación en la propiedad de la misma, caso de Myers y Majluf (1984). Sus teorías buscaron generar modelos que permitieran establecer la estructura adecuada de financiación, según una cantidad determinada de variables. Lo que es claro, es que sus miradas estuvieron enfocadas en la estructura de capital de la empresa, lo que se analizó en este estudio, de manera empírica, con la población descrita, tratando de identificar si existe una relación directa y significativa entre la estructura de capital de la empresa y los resultados que obtuvieron para un periodo determinado. Los datos arrojan, para el caso particular que, aunque hay una relación directa, ésta es insignificante.

\section{Conclusiones.}

Este estudio tuvo como eje central la siguiente pregunta: ¿Existe una relación directa y significativa, entre la estructura de capital de la empresa respecto al resultado financiero del ejercicio de éstas? Para intentar buscar indicios que llevasen a la respuesta a dicha pregunta se planteó trabajar con la información financiera de 2.622 PYME de la localidad de Engativá que reportaron información financiera del año 2017 a la Cámara de Comercio de Bogotá, y que registraron ventas para el año 2017. 
Esta investigación presentó un objetivo general que fue desarrollado a través de los diferentes numerales del presente texto, que fueron estructurados con base a la pregunta de investigación, buscando dar indicios de su respuesta. Respecto al objetivo general que fue "determinar si existe o no una relación directa y significativa, entre la estructura de capital de la empresa respecto al resultado financiero del ejercicio de éstas" se abordó con base la población objeto de estudio (PYME de la localidad de Engativá que reportaron información financiera del año 2017 a la Cámara de Comercio de Bogotá, y que registraron ventas para el año 2017). Se trabajó con un indicador de deuda y un indicador de rendimiento intentando determinar si existe una relación directa y significativa entre la estructura de capital y los resultados financieros obtenidos por la población objeto de estudio previamente descrita.

Partiendo de la base de los datos procesados, respecto al objetivo general es posible asegurar con los resultados de este estudio que no existe una relación directa y significativa entre la estructura de capital de las PYME objeto de estudio respecto al resultado financiero del ejercicio de éstas, para el año 2017. Esto evidenciado en el resultado del coeficiente de correlación de Pearson entre los mencionados indicadores para el total de las 2.622 PYME, que fue de 0,00052299 (cero con cincuenta y dos mil doscientas noventa y nueve cienmillonésimas).

Finalmente, respecto a la pregunta de investigación es necesario resaltar que este estudio, en primera instancia, muestra que no existe una relación directa y significativa entre la estructura de capital de la empresa respecto al resultado financiero del ejercicio. Sin embargo, surgen preguntas tales como: ¿se puede evidenciar una relación directa, significativa, entre la estructura de capital de la empresa, por sector económico, respecto al resultado financiero del ejercicio de éstas? Esta pregunta reconoce que a nivel macroeconómico pueden incidir diferentes variables que afecten el desempeño de empresas de algunos sectores económicos, lo que pudo haber distorsionado los resultados analizados en esta investigación. También queda la siguiente pregunta: ¿se puede evidenciar una relación directa y significativa, entre la estructura de capital de la empresa, según su tamaño, respecto al resultado

financiero del ejercicio de éstas? Esta pregunta teniendo en cuenta que la relación en cuanto a cantidad de las PYME objeto de este estudio fue de 5,5 a 1.

\section{Referencias.}

Barona-Zuluaga, B., \& Rivera-Godoy, J. (2012). Análisis empírico de la financiación de nuevas empresas en Colombia. Innovar, 21(43), 5-18.

Botello, H. (2015). Determinantes del acceso al crédito de las PYMES en Colombia. Ensayos de economía, 46, 135-155.

Cámara de Comercio de Bogotá (2018, 22 de agosto). Preguntas frecuentes. Recuperado de: https://www.ccb.org.co/Preguntas-frecuentes/Camara-de-Comercio-de-Bogota.

Consejo Local de Gestión de Riesgos y Cambio Climático (2017). Caracterización General de Escenarios de Riesgo. Bogotá: Alcaldía Mayor de Bogotá.

Córdoba, M. (2014). Análisis financiero. Bogotá: Ecoe Ediciones.

Decreto 619 de julio 28 de 2000, por el cual se adopta el Plan de Ordenamiento Territorial para Santa Fe de Bogotá, Distrito Capital. Registro Distrital No. 2.197 del 28 de julio de 2000. Bogotá, 28 de julio de 2.000 .

Díaz, H., \& Horngren, C. (2011). Contabilidad para la toma de decisiones. Colombia: Pearson. 
Donaldson, G. (1961). Corporate Debt Capacity: A Study of Corporate Debt Policy and the Determination of Corporate Debt Capacity. Boston: Graduate School of Business Administration, Harvard University.

Fernández, J. (2014). Teoría y práctica de la contabilidad (3a ${ }^{a}$ ed.). Madrid: Ediciones Pirámide.

Gitman, L., \& Zutter, C. (2016). Principio de administración financiera (14 ${ }^{a}$. ed.). Ciudad de México: Pearson.

Herrera, T., De la Hoz, E., \& Vergara, J. (2012). Aplicación de análisis discriminante para evaluar el mejoramiento de los indicadores financieros en las empresas del sector alimento de Barranquilla-Colombia. Ingeniare. Revista Chilena de Ingeniería, 20(3), 320-330.

Ley 590 de 2000 del 10 de Julio de 2000, por la cual se dictan disposiciones para promover el desarrollo de las micro, pequeñas y medianas empresa. Diario Oficial No.44.078 de julio 12 de 2000. Bogotá, 12 de julio de 2000.

Ley 905 de 2004 del 2 de agosto de 2004, por medio de la cual se modifica la Ley 590 de 2000 sobre promoción del desarrollo de la micro, pequeña y mediana empresa colombiana y se dictan otras disposiciones. Diario Oficial No. 45.628 de 2 de agosto de 2004. Bogotá, 2 de agosto de 2004.

Ley 1151 de 2007 del 24 Julio de 2007, por la cual se expide el Plan Nacional de Desarrollo 2006 2010. Diario Oficial No. 46.700 de julio 25 de 2007. Bogotá, 25 de julio de 2007.

Ley 1450 de 2011 del 16 de junio de 2011, por la cual se expide el Plan Nacional de Desarrollo, 20102014. Diario Oficial No. 48.102 de 16 de junio de 2011. Bogotá, 16 de junio de 2011.

Massad, C. (2007). Economía para todos. Santiago de Chile: Editorial e Imprenta Maval LTDA.

Martinez, C., \& Levin, R. (2011). Estadística aplicada. Colombia: Pearson educación.

Modigliani, F., \& Miller, M. (1958). The cost of capital, corporation finance and the theory of investment. The American Economic Review, 48(3), 261-297.

Modigliani, F., \& Miller, M. (1963). Corporate income taxes and the cost of capital. A correction. The American Economic Review, 53(3), 433-443.

Mondragón-Hernández, S. (2011). Marco conceptual de las teorías de la irrelevancia, del trade-off y de la jerarquía de las preferencias. Cuadernos de Contabilidad, 12(30), 165-178.

Myers, S. (1977). Determinants of corporate borrowing. Journal of Financial Economics, 5, 147-175.

Myers, S. (1984). The capital structure puzzle. The Journal of Finance, 39(3), 575-591

Myers, S., \& Majluf, N. (1984). Corporate financing and investment decisions whenfirms have information that investors do not have. Journal of Financial Economics, 13(2), 187-221.

Nieto, V., Timote J., Sánchez, A., \& Villareal S., (2015). La clasificación por tamaño empresarial en Colombia: Historia y limitaciones para una propuesta. Archivos de Economía, 434, 1-48.

O'kean, J. (2013). Economía. Madrid: Mc Graw-Hill Interamericana de España S.L. 
Peña-Barrera, C.R. (2010). Índice de Urbanización Municipal: una aplicación a Bogotá y su teórica "área metropolitana". Territorios, 23, 33-57.

Pesce, G., Esandi, J., Briozzo, A., \& Vigier, H. (2015). Decisiones de financiamiento en pymes: particularidades derivadas del entrelazamiento empresa-propietario. REGE Revista de Gestão, 22(3), 357-380.

Secretaría de Planeación Distrital (2007). UPZ 29, Minuto de Dios. Cartillas pedagógicas del POT. Acuerdos para construir ciudad. Bogotá: Alcaldía Mayor de Bogotá.

Secretaría de Planeación Distrital (2011). 21 monografías de las localidades distrito capital 2011. Diagnóstico de los aspectos físicos, demográficos y socioeconómicos 2011 localidad 10 Engativá. Bogotá: Alcaldía Mayor de Bogotá.

Secretaría de Planeación Distrital (2018a). Proyecciones de Población. Proyecciones de población 2016-2020. Recuperado de: http://www.sdp.gov.co/gestion-estudios-estrategicos/informacioncartografia-y-

estadistica/consulta?combine $=\&$ field_dice_componentes_tid $=326 \&$ field_dice_tema_social_ti $\mathrm{d}=334 \&$ field_dice_tema_territorial_tid=All\&field_dice_tema_economico_tid=All

Secretaría de Planeación Distrital (2018 b). Boletín estadístico \# 5. Dinámica empresarial de BogotáI trimestre 2018. Bogotá: Alcaldía Mayor de Bogotá. 